\title{
TEA, família e escola - O trabalho em conjunto, relação de empatia
}

\author{
TEA, family and school - Working together, empathetic relationship \\ TEA, familia y escuela - Trabajar juntos, relación empática
}

\author{
Aline dos Santos Moreira de Carvalho \\ ORCID: https://orcid.org/0000-0001-9965-9566 \\ Universidad Columbia Del Paraguay, Paraguai \\ E-mail: bioaline2017@yahoo.com \\ Pedro Carlos Pereira \\ ORCID: https://orcid.org/0000-0003-4646-0080 \\ Universidade Federal Rural do Rio de Janeiro, Brasil \\ Universidad Columbia Del Paraguay, Paraguai \\ E-mail: pecape@ig.com.br \\ Camilla Viana de Souza Gonçalo Camilla \\ ORCID: https://orcid.org/0000-0001-5156-4517 \\ Universidad Columbia Del Paraguay, Paraguai \\ E-mail: loramestrado@hotmail.com \\ Gabrielle Oliveira dos Santos Anchieta \\ ORCID: https://orcid.org/0000-0003-1160-569X \\ Universidad Columbia Del Paraguay, Paraguai \\ E-mail: gabbiosantos@yahoo.com
}

\begin{abstract}
Resumo
Cabe ressaltar que o autismo é uma síndrome comportamental que afeta o neurodesenvolvimento caracterizado pelo desenvolvimento atípico que só poderá ser diagnosticada mediante observação de um especialista responsável em detectar o espectro autista nas crianças, que são caracterizados por três sintomas : dificuldade de interação social, déficit quantitativo e qualitativo de comunicação, alteração dos padrões de comportamentos inadequados, como atividades de interesses restritos e estereotipados que são os movimentos de fazer as coisas de forma repetitivas e sem finalidade social. Em sua atualização mais recente - o DSM-5 - o autismo é descrito como um transtorno de desenvolvimento que leva a severos comprometimentos de comunicação social e comportamentos restritivos e repetitivos que tipicamente se inicia nos primeiros anos de vida. O presente artigo traz a discussão para uma efetiva inclusão de pessoas com espectros autistas possam ser inseridos dentro do ambiente educacional em sala de aula de ensino regular e o atendimento educacional especializado por profissionais qualificados de forma a atender as demandas da educação inclusiva, constituindo-se num espaço de aprendizagem e de desenvolvimento da competência social. Os familiares apontam várias fragilidades na inclusão educacional que reforçam a necessidade da interlocução de diversas políticas públicas para a oferta de uma escola inclusiva promotora de desenvolvimento e aprendizagem, tendo em vista que os mesmos possuem uma expectativa na cura quando o estudante adentra os pátios das instituições educacionais.
\end{abstract}

Palavras-chave: Autismo; Inclusão escolar; Família; Ensino.

\begin{abstract}
It is noteworthy that autism is a behavioral syndrome that affects neurodevelopment characterized by atypical development that can only be diagnosed by observation of a specialist responsible for detecting the autistic spectrum in children, which are characterized by three symptoms: difficulty in social interaction, quantitative deficit and qualitative communication, alteration of inappropriate behavior patterns, such as activities of restricted and stereotyped interests, which are movements of doing things repetitively and without social purpose. In its latest update-DSM-5-autism is described as a developmental disorder that leads to severe social communication impairments and restrictive, repetitive behaviors that typically begin early in life. This article brings the discussion for an effective inclusion of people with autistic spectrums that can be inserted into the educational environment in the regular education classroom and the specialized educational service by qualified professionals in order to meet the demands of inclusive education, constituting in a space of learning and development of social competence. Family members point out several weaknesses in educational inclusion that reinforce the need for the dialogue of various public policies to offer an inclusive school that promotes development and learning, given that they have an expectation of healing when the student enters the courtyards of institutions educational.
\end{abstract}

Keywords: Autism; School Inclusion; Family; Teaching. 


\begin{abstract}
Resumen
Cabe destacar que el autismo es un síndrome conductual que afecta al neurodesarrollo caracterizado por un desarrollo atípico que solo puede ser diagnosticado mediante la observación de un especialista encargado de detectar el espectro autista en los niños, los cuales se caracterizan por tres síntomas: dificultad en la interacción social, déficit cuantitativo y comunicación cualitativa, alteración de patrones de conducta inapropiados, tales como actividades con intereses restringidos y estereotipados, que son movimientos de hacer las cosas de manera repetitiva y sin finalidad social. En su última actualización, DSM-5, el autismo se describe como un trastorno del desarrollo que conduce a graves deficiencias en la comunicación social y conductas restrictivas y repetitivas que suelen comenzar temprano en la vida. Este artículo trae la discusión para una inclusión efectiva de las personas con espectros autistas que puedan ser insertadas en el ámbito educativo en el aula de educación regular y el servicio educativo especializado por profesionales calificados con el fin de atender las demandas de la educación inclusiva, constituyéndose en un espacio de aprendizaje y desarrollo de la competencia social. Los familiares señalan varias debilidades en la inclusión educativa que refuerzan la necesidad del diálogo de diversas políticas públicas para ofrecer una escuela inclusiva que promueva el desarrollo y el aprendizaje, dado que tienen una expectativa de curación cuando el alumno ingresa a los patios de las instituciones educativas.
\end{abstract}

Palabras clave: Autismo; Inclusión Escolar; Familia; Enseñanza.

\title{
1. Introdução
}

O Transtorno do Espectro Autista é um transtorno caracterizado pelo comprometimento de muitas habilidades da criança, com prevalência desse em habilidades de interação social, comunicação, comportamento, assim como pelo interesse apresentado em atividades com padrões restritos e repetitivos, o que gera grandes mudanças na dinâmica familiar após o diagnóstico. O enfrentamento por parte da família das crianças com TEA é difícil e penoso pois essas apresentam diferenças no desenvolvimento que interferem diretamente nos desempenhos social e educacional (Mcauliffe et al., 2019 apud Cabral; Falcke; Marim, 2021).

O TEA torna-se um grande desafio para toda a família. Alguns estudos mostraram que é possível superar e enfrentar positivamente, com bons resultados quando existe cooperação, ajuda mútua, coesão e resistência por parte dos familiares (Taboada et al., 2006; Andrade; Teodoro, 2012; Vernhet et al., 2019 apud ibidem), assim como alguns estudos apontaram que existe uma minoria que enfatiza as dificuldades do processo, como a dificuldade frente ao comportamento, a dificuldade em acessar serviços públicos e em ter apoio (saúde, terapêutica, etc) e o difícil consenso para um diagnóstico.

Nesse contexto, a família é um fator determinante no processo inclusivo, mediante sua interação com a escola. Faz-se necessária uma interação verdadeira, principalmente no que tange a informações sobre a comunicação ou comunicações particulares de cada criança, a relação ideal traria a afetividade como caminho.

Nessa perspectiva, o estudo objetiva explanar sobre o TEA e o processo inclusivo, relatando a relação existente entre família e escola, demonstrando a afetividade como um caminho eficaz.

\section{Metodologia}

A metodologia utilizada para a produção deste estudo foi de pesquisa bibliográfica, realizada em meio virtual, com artigos disponível e e-books em sites confiáveis, de conteúdo científico.

A pesquisa bibliográfica, ou de fontes secundárias, abrange toda bibliografia já tornada pública em relação ao tema de estudo, desde publicações avulsas, boletins, jornais, revistas, livros, pesquisas, monografias, teses, material cartográfico etc. [...] Dessa forma, a pesquisa bibliográfica não é mera repetição do que já foi dito ou escrito sobre certo assunto, mas propicia o exame de um tema sob novo enfoque ou abordagem, chegando a conclusões inovadoras (Lakatos, Marconi, 2010, p.166).

Sendo assim, foram selecionados artigos e e-books resultantes de pesquisa em meio virtual, com os descritores: Autismo, TEA, família e escola. 
Artigos que não correlacionavam os três descritores foram excluídos. Após a exclusão, foi feita uma leitura analítica que resultou na fundamentação teórica deste estudo, apresentada em resultados e discussões e subdividida em títulos.

As conclusões, incluindo algumas propostas e as impressões dos autores, encontram-se nas considerações finais.

\section{Resultados e Discussão}

\section{Autismo ou TEA, conceitos e significados}

Atualmente, o Brasil possui dois milhões de autistas, sendo mais trezentas mil somente no estado de São Paulo (Oliveira, 2021).

O autismo não é uma doença singular, mas sim um distúrbio de desenvolvimento complexo, que compreende definições tanto do ponto de vista comportamental, como de múltiplas causas e graus variados de severidade (Onzi; Gomes, 2015 apud Bottan, 2020).

O autismo é caracterizado por ser um transtorno que desenvolve uma condição acentuadamente anormal e prejudicada nas interações sociais, nos processos comunicativos e no comportamento (American Psychiatric Association [APA], 2013 apud Guedes; Tada, 2015), sendo tais características variantes de acordo com a maneira como se manifestam e o grau de severidade do transtorno.

A cartilha Direitos das Pessoas com Autismo (Defensoria Pública Do Estado De São Paulo, 2011 apud Carvalho et al, 2012) descreve o autismo como Transtorno Global do Desenvolvimento (também chamado de Transtorno do Espectro Autista), caracterizado por alterações significativas na comunicação, na interação social e no comportamento da criança, que tem complexas consequências como dificuldades adaptativas que aparecem antes dos três anos de idade, e que podem ser percebidas ainda nos primeiros meses de vida. O TEA tem causas ainda não claramente identificadas, porém já se sabe que o autismo é multifatorial, mais comum em crianças do sexo masculino e independente da etnia, origem geográfica ou situação socioeconômica (Santos; Souza, 2012 apud Carvalho et al., 2012)

Segundo Schmidt, “[...] o TEA é definido como um distúrbio do desenvolvimento neurológico que deve estar presente desde a infância, apresentando déficit nas dimensões sociocomunicativa e comportamental”. (Schmidt, 2013, p. 13 apud Santos; Vieira, online).

E conforme a APA,

[...] as manifestações comportamentais que definem o TEA incluem comprometimentos qualitativos no desenvolvimento sociocomunicativo, bem como a presença de comportamentos estereotipados e de um repertório restrito de interesses e atividades, sendo que os sintomas nessas áreas, quando tomados conjuntamente, devem limitar ou dificultar o funcionamento diário do indivíduo (APA, 2013 apud Zanon et al, 2014, p.25 apud Santos; Vieira, online)

No que diz respeito ao transtorno infantil, apresenta ausência ou dificuldade de comunicação oral (caracterizando o déficit de linguagem), falta de reciprocidade, não socialização, não querer contato com o próximo (características do déficit na interação social) e no aspecto comportamental, apresenta necessidade de rotina e movimentos repetitivos e as estereotipias. (Santos; Vieira, online)

Cunha (2015, p.23 apud Santos; Vieira, online), descreve que utilizar o termo TEA possibilita a compreensão de diversos níveis de transtorno, entre leve, moderado e severo.

Santos e Vieira (online), afirmam que as manifestações do transtorno são percebidos na rotina das crianças, como a dificuldade da comunicação e linguagem, atraso na linguagem oral, pouca ou nenhuma interação social (falta de reciprocidade, dificuldade em socialização e comprometimento no contato com o próximo) e o déficit comportamental. 
"É também comum se observar crianças autistas fascinadas por certos estímulos visuais, como luzes piscando e reflexos de espelho bem como tendo certas aversões ou preferências por gostos, cheiros e texturas específicas [...]" (Silva; Mulick, 2009, p.120 apud Santos; Vieira, online).

Segundo Guedes e Tada (2015), desde a descoberta em 1943, o transtorno tem estado entre discussões e debates a respeito de causas, sintomas e tratamentos. Sabe que possui origem multicausal (Schwartzman, 2011 apud Guedes; Tada, 2015), mas não existem respostas para os fatores especificamente determinantes.

Os cientistas levantam hipóteses de causas psicoafetivas, como um empasse na construção do sujeito subjetivo (Campanário; Pinto, 2011 apud Guedes; Tada, 2015 ou de perturbações internas profundas na relação da criança com o meio (Mecconnel, 2013 apud Guedes; Tada, 2015)). De outro lado, alguns buscam causas neurobiológicas ou geneticamente determinadas, anormalidades cerebrais, alterações neuronais ou translocações cromossômicas. (Tarelho; Assumpção, 2007 apud Guedes; Tada, 2015).

O TEA é um transtorno que apresenta muitas comorbidades. Das comorbidades que atuam no TEA, as de maior índice de ocorrência na população infantil são as patologias gastrointestinais como: a constipação (causando intestino ressecado); a diarreia (que causa desidratação); gastrite que pode progredir para úlcera, uma vez que o indivíduo tem dificuldades na comunicação; esofagite e instabilidade da flora intestinal que desenvolver necessidades maiores de vitaminas e minerais; inadequação na absorção de micronutrientes, de forma a acarretar carências nutricionais (Bianchini, 2014).

As comorbidades no TEA estão diretamente relacionadas ao consumo inadequado de energia, obesidade, desnutrição, ganho ou perda de peso. As alterações na composição e funcionamento da microbiota intestinal, por sua vez, refletem diretamente em sintomas gastrointestinais e disfunções comportamentais e cognitivas (Sharp et al, 2014 apud Magagnin et al, 2019; Yangy et al, 2018 apud Magagnin et al, 2019).

Do ponto de vista psiquiátrico, as comorbidades em crianças com TEA são agravantes, como os distúrbios do sono, o Transtorno Obsessivo Compulsivo (TOC), o Transtorno do Déficit de Atenção com Hiperatividade (TDAH), ansiedade, a estereotipia (ação repetitiva de movimento, postura ou fala), o comportamento infrator e a Deficiência Intelectual (DI), sendo essa a mais frequente e comprometedora do quadro psiquiátrico desenhado pelo TEA. A relação entre o TEA e as comorbidades gastrointestinais e psiquiátricas caracteriza a piora do quadro clínico dessas crianças, uma vez que comprometem o organismo por meio de patologias clínicas e/ou pioram a condição social, cognitiva e comportamental como as psiquiátricas. (Bianchini, 2014).

\section{A legislação inclusiva brasileira}

No Brasil, o atendimento a pessoas com deficiência teve início durante o império, com a criação de duas instituições Instituto dos Meninos Cegos (Instituto Benjamin Constant) e do Instituto dos Surdos-Mudos (Instituto Nacional de Educação de Surdos, INES) (Miranda 2008 apud SANTOS; VIEIRA, online). Porém em 1988, a Constituição Federal assegura que a educação é direito fundamental de todos, firmando um compromisso também de oferecimento de atendimento educacional especializado a pessoas com deficiência.

Dentre a legislação que versa sobre a temática, pode-se destacar a Lei de Diretrizes e Bases da Educação Nacional, Lei no 9.394/96, a Política Nacional da Educação Especial na Perspectiva da Educação Inclusiva (2008), as Diretrizes Nacionais para Educação Especial na Educação Básica (Resolução CNE/CEB No 2, de 11 de setembro de 2001), a lei ${ }^{\circ}$ 13.146/15, que institui a Lei Brasileira de Inclusão da Pessoa com Deficiência e a lei de amparo à pessoa com autismo ( lei $\mathrm{n}^{\circ}$ 12.764/12), considerada uma das mais importantes na inclusão da pessoa com TEA. (Santos; Vieira, online). 
Em 1996, a Lei de Diretrizes e Bases da Educação Nacional (LDBEN), lei nº 9.394/96, atualiza a concepção de ensino para as pessoas com necessidade educacional especial e estabelece um sistema equivalente à educação inclusiva, no capítulo $\mathrm{V}$, sessão da Educação Especial,

Currículos, métodos, técnicas, recursos educativos e organização, específicos para atender às suas necessidades; terminalidade específica para aqueles que não puderem atingir o nível exigido para a conclusão do ensino fundamental, em virtude de suas deficiências [...], professores com especialização adequada em nível médio ou superior, para atendimento especializado, bem como professores do ensino regular capacitados para a integração desses educandos nas classes comuns; educação especial para o trabalho, visando a sua efetiva integração na vida em sociedade, inclusive condições adequadas para os que não revelarem capacidade de inserção no trabalho competitivo, mediante articulação com os órgãos oficiais afins [...] acesso igualitário aos benefícios dos programas sociais suplementares disponíveis para o respectivo nível do ensino regular (Brasil, 1996, p. 19-20 apud Santos; Vieira, online ).

O Brasil, objetivando a educação inclusiva, criou a Política Nacional de Educação Especial na Perspectiva da Educação Inclusiva, portaria n ${ }^{\circ} 555 / 2007$, prorrogada pela portaria 948/2007, “tem como objetivo assegurar a inclusão escolar de alunos com deficiência, transtornos globais do desenvolvimento e altas habilidades/superdotação [...]” (Brasil, 2008, p.15 apud Santos; Vieira, online). Essa cita os transtornos globais do desenvolvimento, que compreende o autismo.

$\mathrm{O}$ acesso e a permanência da criança com necessidade educacional especial no ensino regular são fundamentais para o processo inclusivo, para a aprendizagem e para a própria, como ativa do processo educacional.

Em 2012, no Brasil, a Política Nacional de Proteção dos Direitos da Pessoa com Transtorno do Espectro Autista (intitulada como Lei Berenice Piana) é instituída reconhecendo a importância da pessoa com autismo na sociedade brasileira. A lei alega que o autismo passa a ser considerado como deficiência, e no Artigo 3, a Lei afirma que são direitos das pessoas com TEA:

I - a vida digna, a integridade física e moral, o livre desenvolvimento da personalidade, a segurança e o lazer;

II - a proteção contra qualquer forma de abuso e exploração;

III - o acesso a ações e serviços de saúde, com vistas à atenção integral às suas necessidades de saúde, incluindo: a) o diagnóstico precoce, ainda que não definitivo; b) o atendimento multiprofissional; c) a nutrição adequada e a terapia nutricional; d) os medicamentos; e) informações que auxiliem no diagnóstico e no tratamento;

IV - o acesso: a) à educação e ao ensino profissionalizante; b) à moradia, inclusive à residência protegida; c) ao mercado de trabalho; d) à previdência social e à assistência social. (Brasil, 12.764/12 apud Santos; Vieira, online)

Em 2015 é aprovada a Lei n ${ }^{\circ}$ 13.146/15 que fundamenta e embasa ações inclusivas. A Lei Brasileira de Inclusão da Pessoa com Deficiência (Estatuto da Pessoa com Deficiência), objetiva a igualdade e o exercício dos direitos dessas.

Sobre o direito a educação, o artigo 28 da Lei esclarece que compete ao poder público assegurar, criar, desenvolver, implementar, incentivar, acompanhar e avaliar questões relacionadas a ações inclusivas.

Nesse sentido, são destacadas algumas exposições do artigo:

[...] II - aprimoramento dos sistemas educacionais, visando a garantir condições de acesso, permanência, participação e aprendizagem, por meio da oferta de serviços e de recursos de acessibilidade que eliminem as barreiras e promovam a inclusão plena;

III - projeto pedagógico que institucionalize o atendimento educacional especializado, assim como os demais serviços e adaptações razoáveis, para atender às características dos estudantes com deficiência e garantir o seu pleno acesso ao currículo em condições de igualdade, promovendo a conquista e o exercício de sua autonomia; [...]

VI - pesquisas voltadas para o desenvolvimento de novos métodos e técnicas pedagógicas, de materiais didáticos, de equipamentos e de recursos de tecnologia assistiva; [...].

IX - adoção de medidas de apoio que favoreçam o desenvolvimento dos aspectos linguísticos, culturais, vocacionais e profissionais, levando-se em conta o talento, a criatividade, as habilidades e os interesses do estudante com deficiência; [...] 
XVIII - articulação Inter setorial na implementação de políticas públicas (Brasil, 13.146/15 apud Santos; Vieira, online).

A educação inclusiva está desenvolvendo-se como realidade também na prática, é preciso um olhar sensível por parte de todos, principalmente da família e escola, enquanto instituição social, para que seja trabalhado de forma concreta o processo de inclusão nesse espaço e para que a criança se desenvolva integralmente.

\section{Família e escola, construindo pontes afetivas}

Kubaski (2013 apud Ambrós; Oliveira, 2017 apud Neurosaber, online) menciona os quatro conceitos (presença, participação, aceitação e aprendizagem) desenvolvidos por Tony Booth e Mel Ainscow (2000) que determinam a inclusão plena e efetiva. Segundo os autores:

- Presença: observa se há classes separadas ou outro tipo de segregação, se o aluno participa de práticas em conjunto ou separadamente, qual a frequência escolar, onde está sendo inserido, a correspondência entre o ano escolar e a idade cronológica;

- Participação: observa a qualidade das experiências educacionais e o engajamento do aluno em atividades coletivas;

- Aceitação: observa-se a aceitação por parte dos professores, dos colegas, e outros funcionários, a presença de amizades mais estreitas (melhores amigos), com quem o aluno socializa melhor;

- Aprendizagem: observa tanto o crescimento acadêmico como o desenvolvimento emocional e social, como são realizadas as avaliações, quais são seus principais recursos e dificuldades.

A inclusão efetiva, apesar do aparato legal, acontecerá se tanto a família como a escola estiver engajadas no processo, numa relação de simbiose, ou seja, em trocas de informações com um diálogo aberto e afetivo.

A família participa do processo inclusivo escolar da criança com TEA de maneira muito especial, oferecendo aos educadores informações sobre as formas de comunicação de seus filhos; a família constitui fator determinante para denotação e manutenção assim como para o impedimento do processo inclusivo (Peteers, 1998; Glat, 2003 apud Serra, 2004).

Os pais geralmente estão preocupados com o futuro de seus filhos, apesar de o processo de aprendizagem dessa população ser lenta, devendo ser colocado como prioridade a interação entre pais e equipe escolar (Nilsson, 2003 apud ibidem).

Oliveira (2015), em seu estudo, fez um levantamento de óticas a respeito da relação entre a família e o processo inclusivo escolar. Alguns depoimentos refletem a perspectiva de cada ator do processo:

\section{Professor:}

Sobre a participação da família na escola, o professor regente considera importante, defende que haja uma parceria entre família e escola e que para um bom desenvolvimento do aluno com TEA, é necessário que a família esteja sempre presente:

"A família tem que caminhar lado a lado com a escola. A escola e a família têm que ter essa parceria... Então... a escola e a família falando a mesma linguagem, eu acredito que vai ser melhor para o desenvolvimento da criança... É muito importante a participação da família na escola."

Professor do atendimento educacional especializado

O professor de AEE relatou que os pais geralmente acompanham bem as crianças, participam das reuniões e ele está sempre procurando está em contato com eles, mostrando o que está sendo feito, perguntando sobre os resultados etc. Considera de grande importância essa participação da família, não só dos alunos com TEA, mas da família de todos os alunos: "A escola não pode trabalhar só, tem que ser um trabalho feito família e escola. Porque se só a família faz e a escola não faz ou se só a escola faz e a família não faz, não vai obter um bom resultado".

Responsável

No que diz respeito à participação da família para a aprendizagem dos alunos com TEA, o responsável acredita que deve haver um trabalho em grupo, um ajudando o outro, com um único objetivo: o aprendizado do aluno. "É um trabalho de formiguinha, trabalhar sempre junto para que tenha um bom resultado mais na frente..." Ressalta que é muito importante a família estar sempre presente na escola, acompanhando a vida escolar da criança e, até mesmo, 
cobrando o que é de direito: “A minha participação, eu creio que é muito boa. Quando se faz necessário a minha presença lá ou até mesmo quando não se faz necessário, eu estou lá, cobrando... eu procuro estar sempre na escola, cobrando na medida do possível na questão da parte pedagógica..." Em relação à importância da participação da família no processo de aprendizagem dos alunos com Transtorno do Espectro Autista, todos são unânimes em dizer que é preciso que haja uma parceria entre família e escola. Essas duas instituições precisam fazer um trabalho em conjunto, visando o mesmo objetivo, que é o desenvolvimento do aluno. É bem fundamental a participação ativa dos pais na vida escolar das crianças para que se tenha bons resultados no aprendizado deles. (Serra, 2004, p. 35-36)

Um dos fatores favorecedores para o sucesso escolar das crianças com TEA é a relação estabelecida entre família e escola, a parceria favorece a troca de experiências e no acompanhamento do processo (Chimenes; Santana, 2020).

O processo de inclusão acontece diariamente no processo de interações sociais, a parceria entre família e comunidade escolar, principalmente no sentido de trocas de informações (Serra, 2004).

Martins e Lima (2018 apud Chimenes; Santana, 2020) afirmam que a parceria entre família e escola exerce influência no processo de escolarização dessa população, os professores buscam maiores contatos com a família através de reuniões periódicas, grupos de estudos, palestras, entre outros.

A relação participativa estabelecida entre família e escola é de suma importância para o desenvolvimento das crianças. Para garantir a inclusão escolar de crianças com deficiência, o Atendimento Educacional Especializado recebeu prioridade pelo Plano Nacional de Educação Especial, com a função de organizar, elaborar e identificar os recursos pedagógicos e de acessibilidade para eliminar barreiras em prol da plena participação dos educandos, considerando necessidades particulares, promovendo autonomia e independência no ambiente que está inserido (Brasil, 2011 apud ibidem).

“O atendimento educacional especializado deve envolver a participação da família para garantir pleno acesso e participação dos estudantes, atender às necessidades específicas das pessoas público-alvo da educação especial, e ser realizado em articulação com as demais políticas públicas" (Brasil, 2011, s/p ibidem, p.29).

Na perspectiva inclusiva, a afetividade tem ganhado destaque por ser um caminho eficaz para todos os envolvidos no processo educativo. Sendo assim, cabe ressaltar que a afetividade contribui para a relação estreita que deve ser estabelecida entre família e escola para que a criança com TEA sinta-se acolhida e amada, propiciando o processo inclusivo.

Sobre afetividade, Mattos (2008 apud Rocha online) afirma que as relações afetivas bem desenvolvidas propiciam um ambiente acolhedor e saudável de aprendizagem para o aluno com deficiência.

O domínio afetivo completa o cognitivo pois são complementares. Ainda segundo Mattos (2008), mesmo que um se sobressaia ao outro, se completam.

Ao falar em afetividade, cabe citar Henry Wallon que explica a formação da pessoa em três fatores integrados (afetivo, motor e cognitivo), contrariando a compreensão do ser humano com em partes distintas. Para esse teórico, a afetividade é vista de distintos aspectos e estágios, seja através das características sociais de cada idade (orgânicas, orais e morais) ou pelas condições de maturação do ser humano, como as emoções, sentimentos e paixão. (Wallon apud Almeida, 2012 apud Rocha, online).

A afetividade e a cognição naturalmente possuem bases orgânicas simples que vão adquirindo graus de complexidade de acordo com o contato com o social. "O nascimento da afetividade é anterior à inteligência". Porém, a "inteligência não se desenvolve sem afetividade e vice-versa, pois ambas compõem uma unidade de contrários" (Wallon apud Almeida, 2012, p. 29 e 42 apud Rocha, online).

Segundo Almeida (2012 apud Rocha, online),

A linguagem constitui-se pouco a pouco no meio de sensibilização da criança. Cada vez mais, o diálogo do toque vai tornando-se sem efeito e a comunicação oral torna-se um excelente mecanismo de negociação com a criança. É 
bastante comum perceber-se o quanto o ouvir e o ser ouvido torna-se um imperativo infantil. O elogio transmitido por palavras 1087 substitui o carinho. Com o tempo, as relações afetivas se estendem para o campo do respeito, da admiração. (Almeida, 2012, p. 44 apud Rocha, online).

A afetividade começa no convívio com a família e evolui no convívio com o meio e com a escola, como ambiente necessário para a aprendizagem, precisa oferecer programações que desenvolvam tanto o cognitivo como o afetivo, pois para a inteligência se desenvolver, é necessária a administração dos sentimentos.

Portanto, por intermédio da afetividade são estabelecidos relacionamentos entre a família e a escola para que, juntos, olhem na mesma direção, a inclusão não somente escolar, mas social dos educandos portadores de TEA.

\section{Considerações Finais}

De acordo com o pensamento de Martins e Lima (2018 apud Chimenes; Santana, 2020) afirmam que a parceria entre família e escola exerce influência no processo de escolarização dessa população, os professores buscam maiores contatos com a família através de reuniões periódicas, grupos de estudos, palestras, entre outros.

A família e a escola são os principais contextos de promoção do desenvolvimento social e educacional das crianças. A compreensão da dinâmica das relações familiares é essencial para a compreensão do indivíduo especial, e, para que as propostas pedagógicas e clínicas tenham êxito, é primordial que haja um trabalho paralelo com os familiares, já que as intervenções buscam promover efeitos recíprocos em cada um dos membros. A inclusão da criança com TEA em escola regular pode trazer benefícios ao aluno e aos pais, principalmente quando ocorre parceria efetiva entre escola e família, e isso tem despertado interesse de pesquisadores (Serra, 2010; Cunha, 2009; Camargo e Bosa 2009; Bosa, 2006), porém, essa é uma questão que merece mais pesquisas, estudos e busca por dados empíricos. Assim a escola, por sua vez, precisa ser inclusiva, um lugar acolhedor, onde não haja preconceito, deve ser um espaço de promoção do desenvolvimento das habilidades da criança, os profissionais precisam estar em plena busca de qualificação para que possa dar o devido atendimento dos estudantes com espectro autista e as famílias precisam estar devidamente orientadas.

\section{Referências}

Bianchini, N. C. P. \& Souza, L. A. P. (2014). Autismo e comorbidades: achados atuais e futuras direções de pesquisa. Revista Distúrbios da comunicação. 26(3): 624-6. http://scholar.google.com.br/scholar_url?

Bosa, C. (2006). Autismo: intervenções psicoeducacionais. Revista Brasileira de Psiquiatria, 8(1), 47-53

Bottan, G. P. et al. (2020). Analisar a alimentação de autistas por meio de revisão de literatura. Brazilian Journal of Development. 6(12). https://www.brazilianjournals.com/index.php/BRJD/article/view/21949

Cabral, C. S., Falcke, D., \& Marin, A. H. (2021). Relação Família-Escola-Criança com Transtorno do Espectro Autista: Percepção de Pais e Professoras. Revista brasileira de educação especial, 27 https://www.scielo.br/j/rbee/a/STKcXJNwvxqhGk5QKh8WpLP/

Carvalho et al. Nutrição e autismo: considerações sobre a alimentação do autista. (2012). https://assets.unitpac.com.br/arquivos/revista/51/1.pdf.

Carvalho, K. C. N. de. (2020) Transtorno do Espectro do Autismo: impacto no comportamento Alimentar. Brazilian Journal of health Review. 3(3), 50095023 https://www.brazilianjournals.com/index.php/BJHR/ article/viewFile/10562/8821.

Chimenes, G. M., \& Santana, M. L. da S. (2020). Parceria família e instituição de educação para inclusão de crianças autistas. Revista Anápolis Digital, 11(2), 14-39, https://portaleducacao.anapolis.go.gov.br/revistaanapolisdigital/wp-content/uploads/vol11/2.pdf.

Camargo, S., \& Bosa, C. (2012). Competência social, inclusão escolar e autismo: Um estudo de caso comparativo. Psicologia: Teoria e Prática, 28(3),315324

De Paula, F. M., et al. (2019). Escala de Avaliação do Comportamento Alimentar no Transtorno do Espectro Autista: estudo de validação. Jornal Brasileiro de Psiquiatria,

Dupin, A. A. da S. Q., \& Silva, M. O. (2020). Educação Especial e Legislação brasileira: revisão de literatura. Scientia Vitae. Edição especial. 10(29). 65. revistaifspsr.com/v10n297690.pdf. 
Research, Society and Development, v. 10, n. 15, e136101522820, 2021

(CC BY 4.0) | ISSN 2525-3409 | DOI: http://dx.doi.org/10.33448/rsd-v10i15.22820

Gomes, P. T. M., et al. (2015). Autismo no Brasil, desafios familiares e estratégias de superação: revisão sistemática. Jornal Pediatrico. 91(2), 11-121. institutoneurosaber.com.br/orientacao-familiar-no-autismo-como-proceder.

Guedes, N. P. da S., \& Tada, I. N. C. (2015): A Produção Científica Brasileira sobre Autismo em Psicologia e na Educação. Psicologia: teoria e pesquisa. 31(3), 303-309. www.scielo.br/j/ptp/a/wHQxZZWnLQKtnJS447QfpFb/?format=pdf\&lang=pt.

Lakatos. E. M.: \& Marconi, M. de A. (2010). Fundamentos de Metodologia Científica. (7a ed.) Atlas,

Magagnin, T., et al (2019). Relato de Experiência: Intervenção Multiprofissional sobre Seletividade Alimentar no Transtorno do Espectro Autista. Id on Line Revista. Multidisciplinar e de Psicologia. 13(43), 114-127, https://www.researchgate.net/publication/330266076_Relato de Experiência Intervenção Multiprofissional sobre Seletividade Alimentar no Transtorno do Espectro Autista.

NEUROSABER, Instituto. Autismo: escola e família como aliados na educação. E-book. Pdf. online, p. 1-30. https://rdstation-

static.s3.amazonaws.com/cms\%2Ffiles\%2F29205\%2F1553806178Ebook_-Autismo Escola e familia como aliados na educacao.pdf

Oliveira, C. (2020). Um retrato do autismo no Brasil. Espaço Aberto. Online. http://www.usp.br/espacoaberto/?materia=um-retrato-do-autismo-no-brasil.

Oliveira, A. C. de. (2015). O papel da família no processo de inclusão escolar do aluno com Transtorno do Espectro Autista. Pdf. Brasília, https://bdm.unb.br/bitstream/10483/15065/1/2015_AndreiaCosmeDeOliveira_tcc.pdf.

Oliveira, F. L. (2020). Autismo e inclusão escolar: os desafios da inclusão do aluno autista. Revista Educação Pública, 20(34) https://educacaopublica.cecierj.edu.br/artigos/20/34/joseph-autismo-e-inclusao-escolar-os-desafios-da-inclusao-do-aluno-autista.

Rocha, C. C., et al. (2019). O perfil da população infantil com suspeita de diagnóstico de transtorno do espectro autista atendida por um Centro Especializado em Reabilitação de uma cidade do Sul do Brasil. Physys: revista de saúde coletiva. 29(4). https://www.scielo.br/j/physis/a/nfN4dx9HgDcSXCyjSjqb4SF/?lang=pt.

Rocha, G. S. S., et al. (2019). Análise da seletividade alimentar de crianças com Transtorno do Espectro Autista. REAS/EJCH. 24, e538., https://doi.org/10.25248/reas.e538.2019

Rocha, S. Afetividade no contexto escolar inclusivo. $1^{\circ}$ Seminário Luso-brasileiro de Educação Inclusiva: o ensino e a aprendizagem em discussão. https://ebooks.pucrs.br/edipucrs/anais/i-seminario-luso-brasileiro-de-educacao-inclusiva/assets/artigos/eixo-10/completo-2.pdf

Santos, R. K. dos, Vieira, A. M. E. C. da S. Transtorno do Espectro do Autismo (TEA): do reconhecimento à inclusão no âmbito educacional.pdf. Universidade Federal Rural do Semi-árido. https://periodicos.ufersa.edu.br/index.php/includere/article/view/7413/pdf

Serra, D. C. G. A (2004). inclusão de uma criança com autismo na escola regular: desafios e processos. Pdf. UERJ. http://www.proped.pro.br/teses/teses_pdf/Dayse Carla Genero Serra-ME.pdf. 